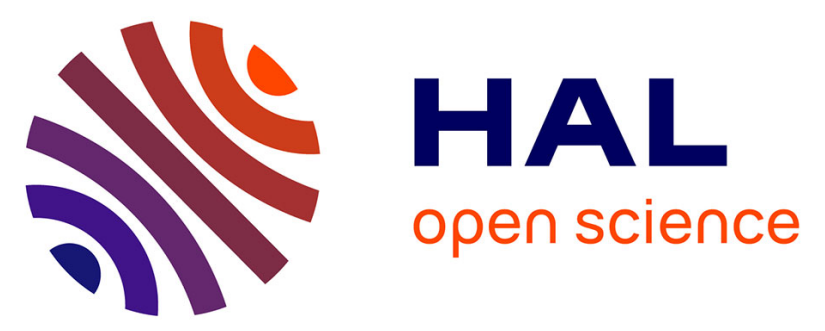

\title{
The effects of secondary electron emission on plasma sheath characteristics and electron transport in an ExB discharge via kinetic simulations
}

\author{
Antoine Tavant, Vivien Croes, Romain Lucken, Trevor Lafleur, Anne
}

Bourdon, Pascal Chabert

\section{To cite this version:}

Antoine Tavant, Vivien Croes, Romain Lucken, Trevor Lafleur, Anne Bourdon, et al.. The effects of secondary electron emission on plasma sheath characteristics and electron transport in an ExB discharge via kinetic simulations. Plasma Sources Science and Technology, 2018, 27 (12), pp.124001. 10.1088/1361-6595/aaeccd . hal-02017799

\section{HAL Id: hal-02017799 \\ https://hal.sorbonne-universite.fr/hal-02017799}

Submitted on 13 Feb 2019

HAL is a multi-disciplinary open access archive for the deposit and dissemination of scientific research documents, whether they are published or not. The documents may come from teaching and research institutions in France or abroad, or from public or private research centers.
L'archive ouverte pluridisciplinaire HAL, est destinée au dépôt et à la diffusion de documents scientifiques de niveau recherche, publiés ou non, émanant des établissements d'enseignement et de recherche français ou étrangers, des laboratoires publics ou privés. 


\title{
The effects of secondary electron emission on plasma sheath characteristics and electron transport in an $E \times B$ discharge via kinetic simulations
}

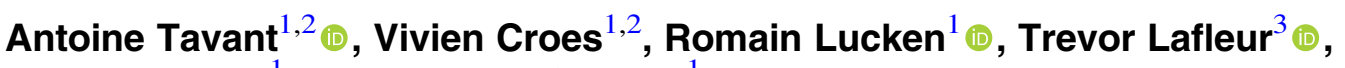 \\ Anne Bourdon ${ }^{1} \odot$ and Pascal Chabert ${ }^{1}$ \\ ${ }^{1}$ Laboratoire de Physique des Plasmas, CNRS, Sorbonne Universités, UPMC Université Paris 6, Université \\ Paris Sud, École Polytechnique, F-91120, Palaiseau, France \\ ${ }^{2}$ Safran Aircraft Engines, Electric Propulsion Unit, F-27208 Vernon, France \\ ${ }^{3}$ PlasmaPotential—Physics Consulting and Research, Canberra ACT 2601, Australia \\ E-mail: antoine.tavant@1pp.polytechnique.fr
}

\begin{abstract}
Hall-effect thrusters, which are electrostatic devices based on an $\mathbf{E} \times \mathbf{B}$ plasma discharge, have successfully been used as satellite propulsion systems for the last few decades. However, the presence of anomalous electron cross-field transport is still poorly understood, and involves complex and strongly coupled mechanisms such as azimuthal electron drift instabilities and intense secondary electron emission (SEE) from the thruster walls. The present work focuses on the relative importance of these two phenomena. We use a 2D particle-in-cell/Monte Carlo collision model configured to simulate the radial-azimuthal directions near the thruster exit plane. A constant radial magnetic field and axial electric field are imposed, and electron drift instabilities are observed in the azimuthal $(\mathbf{E} \times \mathbf{B})$ direction. A simplified SEE model is implemented and an extensive parametric study is performed to directly determine the effect on electron transport. It is found that, for the operating conditions used in our simulations, SEE enhances the near-wall electron mobility by a factor 2 , while reducing the bulk plasma mobility by about $20 \%$ (due to electron cooling). However, the dominant contribution to anomalous electron transport is still observed to be caused by electron drift instabilities driven by the $\mathbf{E} \times \mathbf{B}$ discharge configuration. SEE modifies the electron mobility profile, but the spatially-averaged value remains relatively constant. Three different operating regimes are identified depending on the SEE rate value: two that are stable, and a third which shows an oscillatory behaviour. In addition to electron transport, the kinetic simulations give new insight into the plasma sheath formation at the radial walls, and comparison with typical analytical sheath models demonstrate important differences.
\end{abstract}

Keywords: Hall effect thruster, 2D particle-in-cell simulation, PIC simulation, cross-field electron transport, electron cyclotron drift instability, secondary electron emission, sheath model

\section{Introduction}

The development and understanding of Hall effect thrusters (HETs) is one of the driving forces in the research on $\mathbf{E} \times \mathbf{B}$ plasma discharges. HETs have been used for more than 40 years since the USSR Meteor satellite in 1974 [1] and numerous satellites have been successfully operated using HETs as primary or secondary propulsion system. Nevertheless, the full potential of HETs for space propulsion is not yet attained, as its physics is not clearly understood yet.

The typical HET consists of an annular ceramic channel [2], where an axial electric field $\mathbf{E}$ accelerates the ions and 
creates the thrust. A radial and intense magnetic field $\mathbf{B}$, imposed by an external magnetic circuit, is applied in order to increase the residence time of the electrons, thus increasing the ionization of the propellant, usually xenon. Therefore, properly understanding the cross-field transport of electrons inside the channel would allow the improvement of the HETs. More complete descriptions of the HETs can be found in Morozov et al [3], Adam et al [4], Goebel and Katz [2], Mazouffre [1], Boeuf [5].

Many experimental studies have shown that the electron mobility along the channel axis is much higher than predicted by the classical mobility from drift-diffusion or Bohm diffusion [2, 3, 6-8]. Based on both experimental and numerical studies [4, 9-14], an azimuthal instability raising from the $\mathbf{E} \times \mathbf{B}$ drift of the electrons has been proposed as the main mechanism for the enhanced mobility. Lafleur et al [15] developed a theoretical model of this electron cyclotron drift instability (ECDI) and its effect on the electron mobility. The theory has been successfully confirmed by 1D and 2D PIC simulations [14, 16, 17].

On the other hand, wall material is also known to change the behaviour of the HET discharge [18], and experimental evidences of near-wall transport have been reported [2, 19-21]. The main physical phenomenon proposed to explain this effect from the walls is the intense secondary electron emission (SEE) arising from electron-wall collisions enhancing the electron mobility near the channel walls [3, 20, 21]. However, experiments and simulations [22-27], as well as successfully operating wall-less and magnetic shielded HETs $[28,29]$, show that electron-wall collisions and SEE alone are not sufficient to explain the observed cross-field electron transport. Nevertheless, SEE is expected to significantly alter the plasma behaviour [30], and consequently the study of the enhanced mobility due to ECDI along with the SEE is required to understand the relative importance of these two effects and their coupling.

Various numerical studies of the SEE effects have been performed using unidirectional simulations. A 1D kinetic simulation along the radial direction [31] has demonstrated the importance of SEE on the creation of electron beams between the walls. Fluid simulations [32] highlighted the importance of SEE and electron backscattering for near-wall conductivity. However, those simulations did not model the azimuthal direction, and thus were unable to highlight the effects of the ECDI.

Hèron and Adam [33] used a 2D-3V PIC simulation in order to model the $r-\theta$ plane and study the coupling between SEE effects and ECDI effects on the HET operations. They have shown that SEE does not reduce the ECDI. However their model allows to simulate only the first microseconds of the discharge, and not to study a steady state. Finally, Taccogna et al [34] used a more complex 3D-3V PIC simulation to successfully study these effects. However, the authors used a scaling factor in order to decrease the size of the simulation domain, which affects the instability growth rate and amplitude, hence the electron transport [17]. Consequently, the study of the coupling between SEE effects and the self-consistently simulated azimuthal instability needs to be pursued. In particular, this study should be performed on long time scales and without any scaling.

As the work of Hèron and Adam [33] is very similar to the work presented here, a deeper discussion is warrented. Three main points of interest can be highlighted. First, the model used in [33] did not take into account axial plasma losses, and as a result, no steady state discharge could be reached. Instead the electron temperature, and hence the SEE rate, continuously increased with time [33, figures 17 and 18]. In this work we have proposed a different simulation model that approximately accounts for axial losses, and consequently, allows for the formation of a steady state plasma. Secondly, [33] used radial boundary conditions that mimic the floating nature of dielectric walls. In the present work we have chosen not to explicitly model a dielectric, but we still nonetheless model floating radial boundaries where the net current to the walls is zero; as occurs in real HETs. Thus no major differences are expected from this simplification. Lastly, we have neglected the curvature of the thruster channel. The authors in [33] have shown that the SEE process depends to some extent on the curvature as the radii of the inner and outer surfaces of the channel are different. Here we have again chosen to neglect this effect so as to focus just on the coupling between SEE and the ECDI, and to more clearly understand the fundamental physics involved.

Meanwhile, fluid simulations [7, 32, 35] model the plasma-wall interaction using a sheath model with SEE given by fluid theory [30, 36, 37]. Experimental measurements of the sheath with SEE have been performed in simple conditions [38]. However, to the best of our knowledge, far too little attention has been paid on the validation of these sheath models in the condition of the HET.

The aim of the present investigation is to quantify by a parametric study the relative importance of the ECDI and the SEE on the electron mobility in HETs. This is achieved by using a 2D-3V PIC simulation code able to reach a steady state by setting a finite length in the third dimension, as described in our previous study [14]. A simple SEE model [32] is implemented in order to investigate the importance of ECDI compared to SEE effects. The electron cross-field transport measured in the simulation is compared to the kinetic model of Lafleur et al [15] over a wide range of values of the re-emission rate.

We will firstly describe the different numerical and physical models used in this work in section 2. Then sections 3 and 4 will describe the results obtained from the simulations and the comparisons with the predicted values concerning electron mobility, SEE rates and sheath characteristics.

\section{Models}

\section{1. $2 D$ PIC simulation of a HET}

The results presented have been obtained thanks to a $2 \mathrm{D}-3 \mathrm{~V}$ Particle-in-cell/Monte-Carlo collisions (PIC/MCC) simulation code: LPPic. Details concerning the models and 


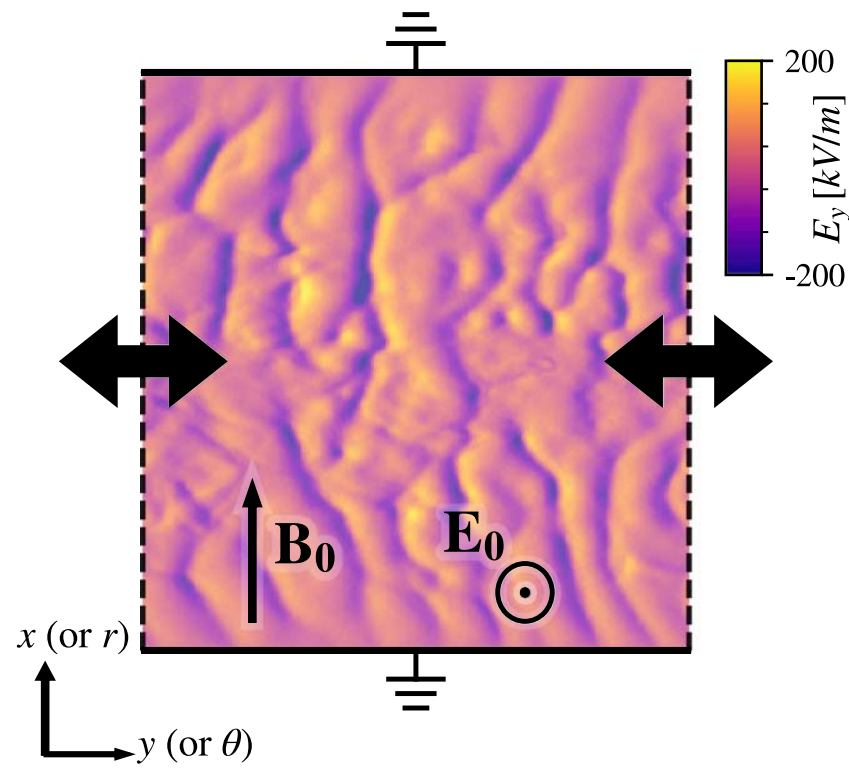

Figure 1. Schematic representation of the 2D simulation domain. Overlaid is a snapshot of the azimuthal electric field $E_{y}$ taken at $t=10 \mu \mathrm{s}$. Lengths used are: $L_{y}=10 \mathrm{~mm}$, and $L_{x}=20 \mathrm{~mm}$.

validations (benchmark, convergence and numerical heating) used for LPPic can be found in Croes et al [14]. We simply recall that LPPic simulates the $r-\theta$ plane without any scaling method and does not consider the thruster curvature.

We note that the curvature in a real thruster can induce asymmetries in the plasma radial profile, as well as the relative magnitude of plasma-wall interactions [33, 39]. Hence, the dispersion relation of the ECDI instability can be different in the regions near the inner and outer channel radii. While this may affect the results obtained, we have specifically chosen to neglect the curvature so as to better focus on the fundamental coupling of the SEE and the ECDI.

The bi-dimentionnal simulation domain uses a Cartesian coordinate system. The $O x$ direction corresponds to the 'radial' direction of the thruster $(r)$, and the $O y$ direction corresponds to the 'azimuthal' direction $(\theta)$. As represented in figure 1, we impose a uniform magnetic field $\mathbf{B}_{0}$ along the 'radial' direction, and a perpendicular electric field $\mathbf{E}_{0}$ in the axial direction, normal to the simulation domain. Hence, the $\mathbf{E}_{0} \times \mathbf{B}_{0}$ direction corresponds to the 'azimuthal' $O y$ direction. The electric field in the simulation domain $\mathbf{E}(x, y)$ is computed by solving Poisson's equation. The simulation domain is closed by walls in the $O x$ direction, while periodic boundaries are used along the $O y$ axis. The walls absorb all incident particles and have a fixed potential $\phi=0 \mathrm{~V}$. Although imposing such a potential may appear artificial, it is worth noting that the thruster anode and cathode are not modelled in the simulation, so the actual potential chosen on the radial boundaries is simply a convenient reference potential used in the solution of Poisson's equation. Due to the symmetric nature of the simulation domain, no net electron and ion current can flow to the radial boundaries, and thus they effectively represent floating walls. This is similar to the floating nature of more realistic dielectric walls, except that the net current must only be zero over the entire length of
Table 1. Standard operating and numerical parameters used in the 2D PIC simulations of an HET. The simulation results are given as representative values.

\begin{tabular}{lcc}
\hline Physical parameter & Value & Unit \\
\hline Gas & Xenon & - \\
$L_{x} \times L_{y} \times L_{z}$ & $2.0 \times 0.5 \times 1.0$ & $\left(\mathrm{~cm}^{3)}\right.$ \\
$B_{0}$ & 200 & $(\mathrm{G})$ \\
$E_{0}$ & $2 \times 10^{4}$ & $\left(\mathrm{Vm}^{-1}\right)$ \\
$n_{0}$ & $3 \times 10^{17}$ & $\left(\mathrm{~m}^{-3}\right)$ \\
$\mathrm{T}_{e, 0}$ & 5.0 & $(\mathrm{~V})$ \\
$T_{i, 0}$ & 0.1 & $(\mathrm{~V})$ \\
$T_{\text {see }}$ & 1.0 & $(\mathrm{~V})$ \\
$P_{n}$ & 1.0 & $\left(\mathrm{mTorr}^{-3}\right)$ \\
$T_{n}$ & 300 & $(\mathrm{~K})$ \\
$n_{g}$ & $3.22 \times 10^{19}$ & $\left(\mathrm{~m}^{-3}\right)$ \\
\hline Simulation parameter & Value & Unit \\
\hline$\Delta t$ & $4 \times 10^{-12}$ & $(\mathrm{~s})$ \\
$\Delta x=\Delta y=\Delta z$ & $2 \times 10^{-5}$ & $(\mathrm{~m})$ \\
$N / N G$ & 80 & $(\mathrm{part} / \mathrm{cell})$ \\
\hline Simulation result & Value & Unit \\
\hline $\mathrm{T}_{e}$ & 40 & $(\mathrm{~V})$ \\
$\omega_{p e}$ & $3.1 \times 10^{10}$ & $(\mathrm{rad} \mathrm{s})$ \\
$\omega_{c e}$ & $3.5 \times 10^{9}$ & $(\mathrm{rad} \mathrm{s})$ \\
$r_{L e}$ & $6 \times 10^{-4}$ & $(\mathrm{~m})$ \\
\hline
\end{tabular}

the wall, whereas with dielectric walls the net current is zero at each grid point along the wall. As the 'azimuthal' simulation distance is relatively small, this is not expected to play any significant role, and has been explicitly chosen so as to remove the added complexity of dielectrics. This allows a more transparent and focussed understanding of the coupling between the SEE effect, and the ECDI.

The $O z$ direction, normal to the $(O x, O y)$ simulation plane is closed by fixing a physical length $L_{z}$ in order to reach a steady state. Particles crossing the lower and upper simulation domain boundaries are re-injected cold on the other side. Details and consequences of this finite length have already been described for a similar 1D case [17], and extensively discussed for the 2D case [14, 40].

Unless otherwise stated, the operating conditions and numerical parameters used in order to run the simulations are listed in table 1 . They have been chosen in order to respect the PIC conditions [41]. Under these conditions, it requires approximately $32 \mathrm{~h}$ with $360 \mathrm{CPUs}$ to simulate $2.5 \times 10^{6}$ time-steps, which corresponds to approximately $10 \mu \mathrm{s}$ of physical time.

In table $1, T_{i, 0}$ and $\mathrm{T}_{e, 0}$ are respectively the ion and electron temperatures at initialization, $T_{\text {see }}$ is the temperature of emission of secondary electrons, $n_{0}$ is the initial plasma density, and $P_{n}, T_{n}$ and $n_{g}$ are respectively the neutral pressure, temperature, and density. The simulation parameters are $\Delta t$ the time step, $\Delta x$ the cell length, and $N / N G$ the average number of particles/cell. Statistical convergence has been tested by increasing the number of particles/cell up to 340 , 


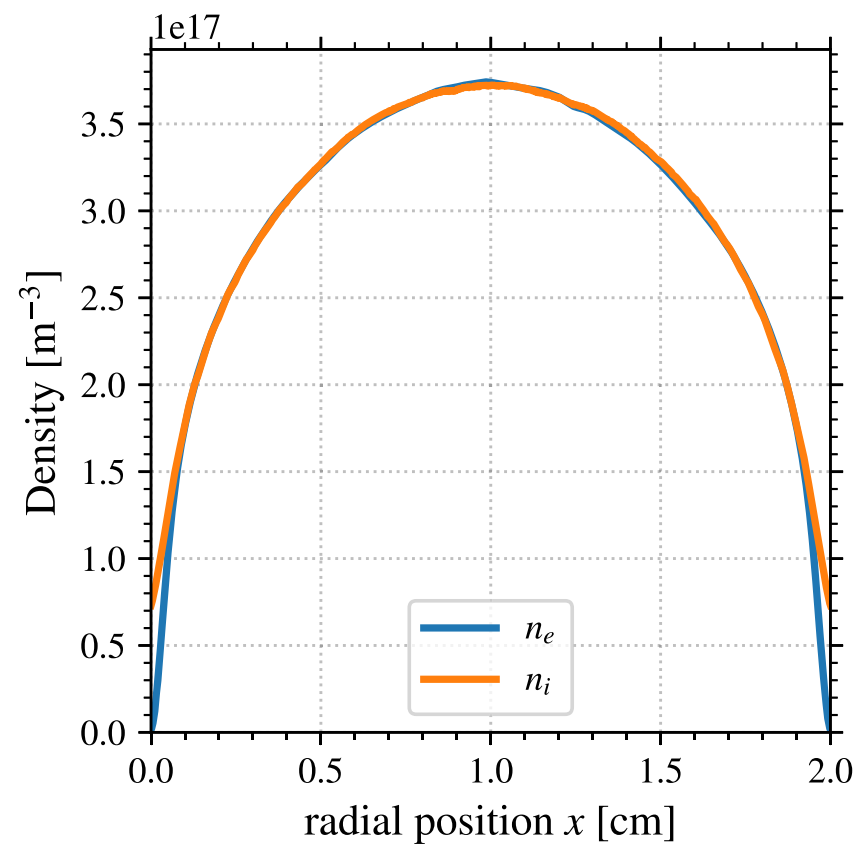

Figure 2. Radial profiles of the electron density $n_{e}$ and the ion density $n_{i}$ averaged in the azimuthal direction for the same parameters as figure 1 .

although little effect on the plasma discharge is observed compared with 80 particles/cell. The average electron temperature $\mathrm{T}_{e}$, plasma frequency $\omega_{p e}$, electron gyrofrequency $\omega_{c e}$ and electron Larmor radius $r_{L e}$ are given as representative values for the simulations.

These values are also close to those used in the previous 1D PIC [17, 42] and 2D PIC simulations [14], ensuring comparability. As observed in Lafleur et al [17], Croes et al [14], using the operating conditions given by table 1 , the wavelength of the ECDI is expected to be about $1.8 \mathrm{~mm}$.

Figure 1 presents the azimuthal electric field with an azimuthal length of $L_{y}=10 \mathrm{~mm}$ with no SEE. The instability is clearly seen and the dominant wave length obtained by Fourier transform is $\lambda=1.44 \mathrm{~mm}$. Reducing the azimuthal length to $L_{y}=5 \mathrm{~mm}$ did not alter the ECDI characteristics i.e. its frequency, wavelength, and amplitude. Hence, and in order to reduce the simulation time of the study, the length $L_{y}=5 \mathrm{~mm}$ is used in the rest of the study.

Figure 2 illustrates the radial density profiles of the electrons and ions. We can see that the centre of the simulation is quasi-neutral, and that the sheath thickness is of the order of the millimetre.

\subsection{SEE model}

The system detailed in section 2.1 has already been tested and studied in Croes et al [14] without SEE. In the present work, a simple linear model $[32,33,43,44]$ is implemented for the SEE process. In this model, the incident electron kinetic energy is used to estimate the re-emission probability as

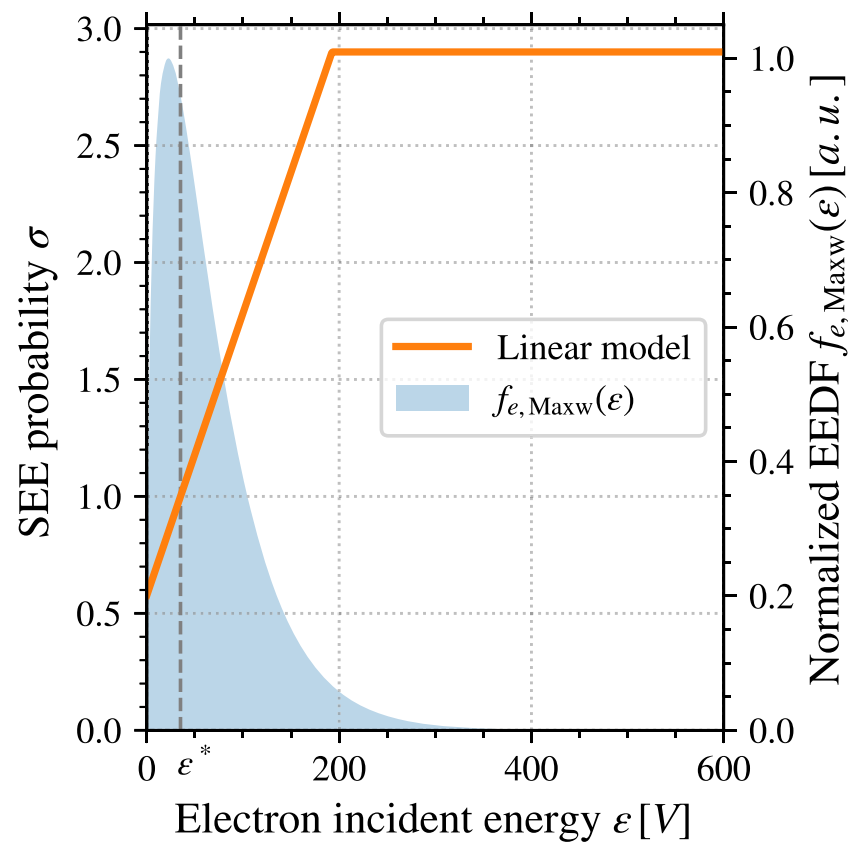

Figure 3. Representation of the SEE model described in section 2.2. Overlaid is a Maxwellian electron energy distribution function of temperature $\mathrm{T}_{e}=45 \mathrm{~V}$. The dashed line corresponds to $\epsilon=\epsilon^{*}=35.04 \mathrm{~V}$.

illustrated in figure 3 and given by:

$$
\sigma(\epsilon)=\min \left(\sigma_{\max }, \sigma_{0}+\left(1-\sigma_{0}\right) \frac{\epsilon}{\epsilon^{*}}\right),
$$

where $\epsilon=\frac{1}{2} m_{e}\left|\mathbf{v}_{\mathrm{e}}\right|^{2}$ is the electron kinetic energy with $m_{e}$ the electron mass and $\mathbf{v}_{\mathbf{e}}$ the electron velocity vector, $\epsilon^{*}$ and $\sigma_{0}$ two parameters, and $\sigma_{\max }$ the maximum re-emission probability.

In this work we chose $\sigma_{\max }=2.9, \sigma_{0}=0.5$, and we varied $\epsilon^{*}$ between 4 and $200 \mathrm{~V}$. This allows us to compare the plasma discharge behaviour with various re-emission strengths from the walls. For example, $\epsilon^{*}=50 \mathrm{~V}$ is a good approximation for $\mathrm{BnSiO}_{2}$ walls [33]. $\mathrm{Al}_{2} \mathrm{O}_{3}$, a more emissive material, would be better modelled with $\epsilon^{*}=17 \mathrm{~V}$, while Graphite, less emissive, is better modelled with $\epsilon^{*}=280$ [18].

As observed in figure 3 , the probability of re-emission can be higher than 1 which means that a single incident electron can extract more than one secondary electron from the wall. In figure 3, a Maxwellian electron energy distribution function (EEDF) of temperature $\mathrm{T}_{e}=45 \mathrm{~V}$ is also plotted to compare the relative position of SEE probability and of the EEDF for a typical energy of electrons in HETs:

$$
f_{e, \operatorname{Maxw}}(\epsilon)=\frac{2}{\sqrt{\pi}}\left(\frac{\epsilon}{\mathrm{T}_{e}}\right)^{1 / 2} \exp \left(-\frac{\epsilon}{\mathrm{T}_{e}}\right)
$$

We can observe that the saturation of the linear model appears in the high energy tail of the EEDF, therefore involving only a small part of the electrons. The secondary electrons are reinjected with a temperature $T_{\text {see }}$ following a Maxwellian distribution in the two directions parallel to the wall, and a Maxwellian flux in the direction normal to the wall. 
The SEE rate is defined by integrating the emission probability over the half distribution function, giving:

$$
\bar{\sigma}\left(f_{e}\right)=\frac{\iiint_{\Omega} v_{x} \sigma\left(\mathbf{v}_{\mathbf{e}}\right) f_{e}\left(\mathbf{v}_{\mathbf{e}}\right) \mathrm{d}^{3} v}{\iiint_{\Omega} v_{x} f_{e}\left(\mathbf{v}_{\mathbf{e}}\right) \mathrm{d}^{3} v}
$$

with $v_{x}$ the radial components of $\mathbf{v}_{\mathbf{e}}, \Omega$ is the half space $\left\{\mathbf{v}_{\mathbf{e}}, \mathbf{v}_{\mathbf{e}} \cdot \mathbf{n}>0\right\}$, with $\mathbf{n}$ a unit vector towards the wall, and $f_{e}\left(\mathbf{v}_{\mathbf{e}}\right)$ is the electron velocity distribution function. Hence $\mathbf{n} \equiv+\mathbf{e}_{x}$ for the upper wall, and $\mathbf{n} \equiv-\mathbf{e}_{x}$ for the lower. Using equation (1) and assuming a Maxwellian electron distribution, we can calculate $\bar{\sigma}_{\mathrm{Maxw}}\left(T_{e}\right)$ as detailed in the appendix. Neglecting the saturation at $\sigma_{\max }$ of equations (1) and (3) reduces to:

$$
\bar{\sigma}_{\text {Maxw }}\left(T_{e}\right)=\sigma_{0}+\left(1-\sigma_{0}\right) \frac{2 T_{e}}{\epsilon^{*}} .
$$

In the simulations, the measured SEE rate, $\bar{\sigma}_{\mathrm{PIC}}$, is assessed using the discretized form of equation (3), that is to say by counting the number of electrons re-emitted by the walls, and dividing it by the number of electrons hitting the wall. It is important to note that even if an individual electron can emit more than one secondary electron, $\bar{\sigma}$ has to be smaller than one to sustain the discharge.

\subsection{Sheath model}

The sheath model featuring SEE processes has been historically studied by Hobbs and Wesson [30], but is still an active research topic nowadays [37]. The sheath is often considered to be collision-less and isothermal, while the plasma is composed of hot Maxwellian electrons and cold ions. A third population of electron-induced secondary electrons is also present in the sheath, and the re-emission rate $\bar{\sigma}$ is assumed to be constant. The SEE process modifies the potential drop in the sheath as [30]:

$$
\Delta \phi_{\text {sheath }}=\mathrm{T}_{e \|} \ln \left[(1-\bar{\sigma}) \sqrt{\frac{m_{i}}{2 \pi m_{e}}}\right]
$$

with $m_{i}$ the ion mass, and $\mathrm{T}_{e \|}$ the electron temperature in the direction parallel to the magnetic field, thus normal to the walls. Adding a pre-sheath drop of $\mathrm{T}_{e \|} / 2$ [36], the total potential drop to the wall is:

$$
\Delta \phi=\Delta \phi_{\text {sheath }}+\frac{\mathrm{T}_{e \|}}{2}=\mathrm{T}_{e \|}\left[\frac{1}{2}+\ln \left[(1-\bar{\sigma}) \sqrt{\frac{m_{i}}{2 \pi m_{e}}}\right]\right] .
$$

At this point, it is important to note that the temperature is defined in our work from the kinetic definition:

$$
T_{e}=\frac{m_{e}}{3|q| n_{e}} \iiint_{-\infty}^{+\infty}\left|\mathbf{v}_{\mathbf{e}}-\mathbf{U}\right|^{2} f_{e}\left(\mathbf{v}_{\mathbf{e}}\right) \mathrm{d}^{3} v
$$

with $\mathbf{U}$ the mean velocity vector, $q$ the electron charge, and $n_{e}$ the electron density. This definition allows us to extend the definition of $\mathrm{T}_{e}$ for non-Maxwellian populations and to use it directly in the PIC simulation. Similarly, the parallel electron temperature used in equation (5) can be expressed by integrating $f_{e}\left(\mathbf{v}_{\mathbf{e}}\right)$ along the radial direction:

$$
T_{e \|}=\frac{m_{e}}{|q| n_{e}} \iiint_{-\infty}^{+\infty}\left(v_{x}-U_{x}\right)^{2} f_{e}\left(\mathbf{v}_{\mathbf{e}}\right) d^{3} v
$$

with $U_{x}$ the radial components of $\mathbf{U}$.

We can easily observe that equation (5) predicts a critical value at which $\Delta \phi_{\text {sheath }}=0$ :

$$
\bar{\sigma}_{\mathrm{cr}}=1-\sqrt{\frac{2 \pi m_{e}}{m_{i}}} .
$$

For xenon, we obtain $\bar{\sigma}_{\mathrm{cr}} \simeq 0.985$ [2].

\subsection{Mobility}

The electron mobility in the transverse direction of the simulation domain is defined, and calculated, as:

$$
\mu=\frac{U_{z}}{E_{0}}
$$

with $U_{z}=\left\langle v_{e, z}\right\rangle$ the average electron velocity in the axial direction. In the PIC simulation, equation (10) is used to calculate $\mu_{\mathrm{PIC}}$.

The classical drift-diffusion theory of the mobility transverse to a magnetic field predicts a mobility $\mu_{\text {classical }}$ expressed as [6]:

$$
\mu_{\text {classical }}=\frac{|q|}{m_{e}} \frac{\nu_{m}}{\nu_{m}^{2}+\omega_{c e}^{2}},
$$

with $\omega_{c e}=|q| B_{0} / m_{e}$ the electron cyclotron frequency, and $\nu_{m}$ the electron-neutral momentum transfer collision frequency.

Lafleur et al $[15,17]$ have shown that anomalous transport due to an enhanced electron-ion friction force induced by the ECDI may be modelled by the following effective mobility:

$$
\mu_{\text {eff }}=\mu_{\text {classical }}\left(1-\frac{\omega_{c e}}{\nu_{m}} \frac{\left\langle\delta n_{e} \delta E_{y}\right\rangle}{n_{0} E_{0}}\right)
$$

with $n_{0}$ the mean electron density, $\delta n_{e}$ the fluctuation of the electron density and $\delta E_{y}$ the fluctuation of the azimuthal electric field. In our simulation set-up and assuming that the saturation of the instability is due to ion trapping, the mobility can be expressed as:

$$
\mu_{\mathrm{eff}}^{\mathrm{sat}}=\mu_{\text {classical }}\left(1+\frac{\omega_{c e}}{\nu_{m}} \frac{R_{e i}}{|q| n_{0} E_{0}}\right),
$$

where

$$
\begin{aligned}
R_{e i} & =-|q|\left\langle\delta n_{e} \delta E_{y}\right\rangle_{\text {saturation }} \\
& =\frac{\left|q \nabla \cdot\left(n_{e} \mathrm{~T}_{e} v_{i}\right)\right|}{4 \sqrt{6} c_{s}} \approx \frac{|q| n_{e} \mathrm{~T}_{e} v_{i z}}{4 \sqrt{6} c_{s} L_{z}}
\end{aligned}
$$

is the saturated electron-ion friction force. In equation (14), $\mathbf{v}_{i}$ is the local ion drift velocity, $c_{s}=\left(|q| \mathrm{T}_{e} / m_{i}\right)^{1 / 2}$ is the Bohm speed, and the spatial derivative has been approximated across the axial simulation direction and it was assumed that there are no derivatives in the radial and azimuthal directions. 
The ion outlet velocity along $(\mathrm{Oz})$ in the simulation is:

$$
v_{i z}=\left(\frac{2|q| V_{z}}{m_{i}}\right)^{1 / 2}
$$

with $V_{z}=E_{0} L_{z}$ the total potential difference in the axial direction. Neglecting the collision frequency against the cyclotron frequency, equation (13) can be simplified to:

$$
\mu_{\mathrm{eff}}^{\text {sat }}=\frac{\left(T_{e} / V_{z}\right)^{1 / 2}}{4 \sqrt{3} B_{0}} .
$$

Equation (16) shows that for the simple 2D PIC geometry used here, the enhanced mobility is only a function of the electron temperature. However, this is not true in general. We can note that $\mu_{\text {eff }}$ can be estimated at each grid-point of the simulation domain, similarly to $\mu_{\mathrm{PIC}}$. On the contrary $\mu_{\mathrm{eff}}^{\text {sat }}$ can only be globally calculated over the whole simulation domain.

In section 4 , comparisons between $\mu_{\text {PIC }}$ the measured mobility in the simulations and the predicted values of $\mu_{\text {eff }}$ and $\mu_{\text {eff }}^{\text {sat }}$ will be presented to quantify the relative contributions of the ECDI and SEE to the electron cross-field transport.

\section{Results}

A parametric study is conducted using the SEE model detailed in section 2.2 with $\sigma_{0}=0.5$ and $\sigma_{\max }=2.9$ while the value of $\epsilon^{*}$ is varied from 4 to $200 \mathrm{~V}$. For every simulation presented hereafter, we observe azimuthal oscillations as shown in figure 1. The simulations reach a steady state, characterized by stable oscillations, in less than $2 \mu \mathrm{s}$ in every case. The ECDI characteristics are consistent with previous studies [14], validating the kinetic theory of Lafleur et al [15]. The physical values presented in this section are averaged in space over the azimuthal direction and in time between $t=5 \mu \mathrm{s}$ and $t=10 \mu \mathrm{s}$.

Figure 4 compares the value of the measured SEE rate $\bar{\sigma}_{\text {PIC }}$ obtained from the PIC simulations with $\bar{\sigma}_{\text {Maxw }}$ from equation (4) using $\mathrm{T}_{e}$ measured in the whole simulation domain using equation (7). We can see that in the PIC simulations, $\bar{\sigma}_{\text {PIC }}$ decreases monotonically from 1 for low $\epsilon^{*}$ to a value close to $\sigma_{0}$, as expected, for high $\epsilon^{*}$. This convergence to 1 has already been observed and commented by Sydorenko et al [45]. For $\epsilon^{*} \leqslant 40 \mathrm{~V}$ we observe that $\bar{\sigma}_{\mathrm{PIC}}>\bar{\sigma}_{\mathrm{cr}}$. It corresponds to a regime of non-monotonic potential profile. Indeed, when the SEE is too intense the charge density close to the wall becomes negative to the point where the sign of the electric field changes. Hence, the plasma potential profile forms a well close to the wall, as already observed in Sydorenko et al [44]. In this low $\epsilon^{*}$ limit, the theory of a standard monotonic sheath is not valid.

However, equation (4) is expected to predict well $\bar{\sigma}$ for $\epsilon^{*}>40 \mathrm{~V}$. Yet, figure 4 shows that the predicted values of $\bar{\sigma}_{\text {Maxw }}$ using equation (4) and the electron temperature measured in the simulations are much higher than the PIC simulation results.

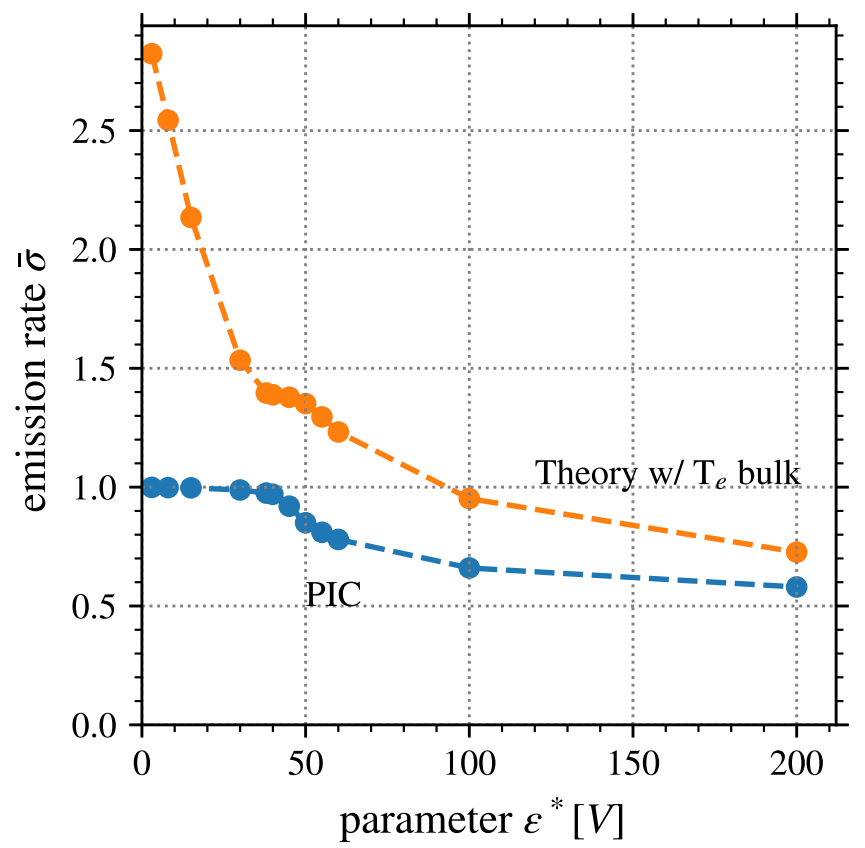

Figure 4. SEE rates averaged over time and space over the whole computational domain as a function of the re-emission parameter $\epsilon^{*}$. Comparison of $\bar{\sigma}_{\text {PIC }}$ derived from the PIC simulations and $\bar{\sigma}_{\text {Maxw }}$, the Maxwellian theory of equation (4) with $T_{e}$ measured in the PIC simulations.

In order to check the sheath model, figure 5(a) presents as a function of the SEE rate measured in the PIC simulations $\bar{\sigma}_{\mathrm{PIC}}$, the evolution of the total potential drop $\Delta \phi$ measured between the wall and the centre of the domain, normalized to the electron temperature $\mathrm{T}_{e}$ given in figure 5(b). It is compared to equation (6), normalized to $T_{e}$ as well. In PIC simulations, the electron temperature shown in figure $5(\mathrm{~b})$ decreases monotonically with the SEE rate $\bar{\sigma}$, from $45 \mathrm{~V}$ for low emission to around $30 \mathrm{~V}$ for high emission. Figure 5(a) shows that the value of $\Delta \phi / T_{e}$ derived from PIC simulations follows the same trend as the theoretical value from equation (6), that is a monotonic decrease with $\bar{\sigma}$. The PIC results seem to show a transition between two different regimes around $\bar{\sigma}_{\mathrm{PIC}}=0.8$. This is discussed further in section 3.3.

However, on figure 5(a), the values differ quite significantly for both the high and low limits. Indeed, for high $\bar{\sigma}$ the expected potential drops to negative value, while we observe that $\Delta \phi / \mathrm{T}_{e}$ tends towards 1 in the simulations. This difference can be explained by the fact that the model described in section 2.3 is only valid for a monotonic sheath, while for high SEE rate $\left(\epsilon^{*}<40 \mathrm{~V}\right)$ we observe a potential well near the wall as previously discussed. Hobbs and Wesson [30] showed that under these conditions of high emission, the total potential drop to the wall goes down to $\mathrm{T}_{e}$ and not to zero or below, as we observe in figure 5(a).

For low values of $\bar{\sigma}$, the measured sheath drop is lower than the value predicted by almost one third. This observation is more surprising, as the sheath is monotonic for these parameters, and should hence be well predicted.

Figures 5 and 4 show that the sheath model proposed in section 2 is not satisfactory when confronted to the PIC 

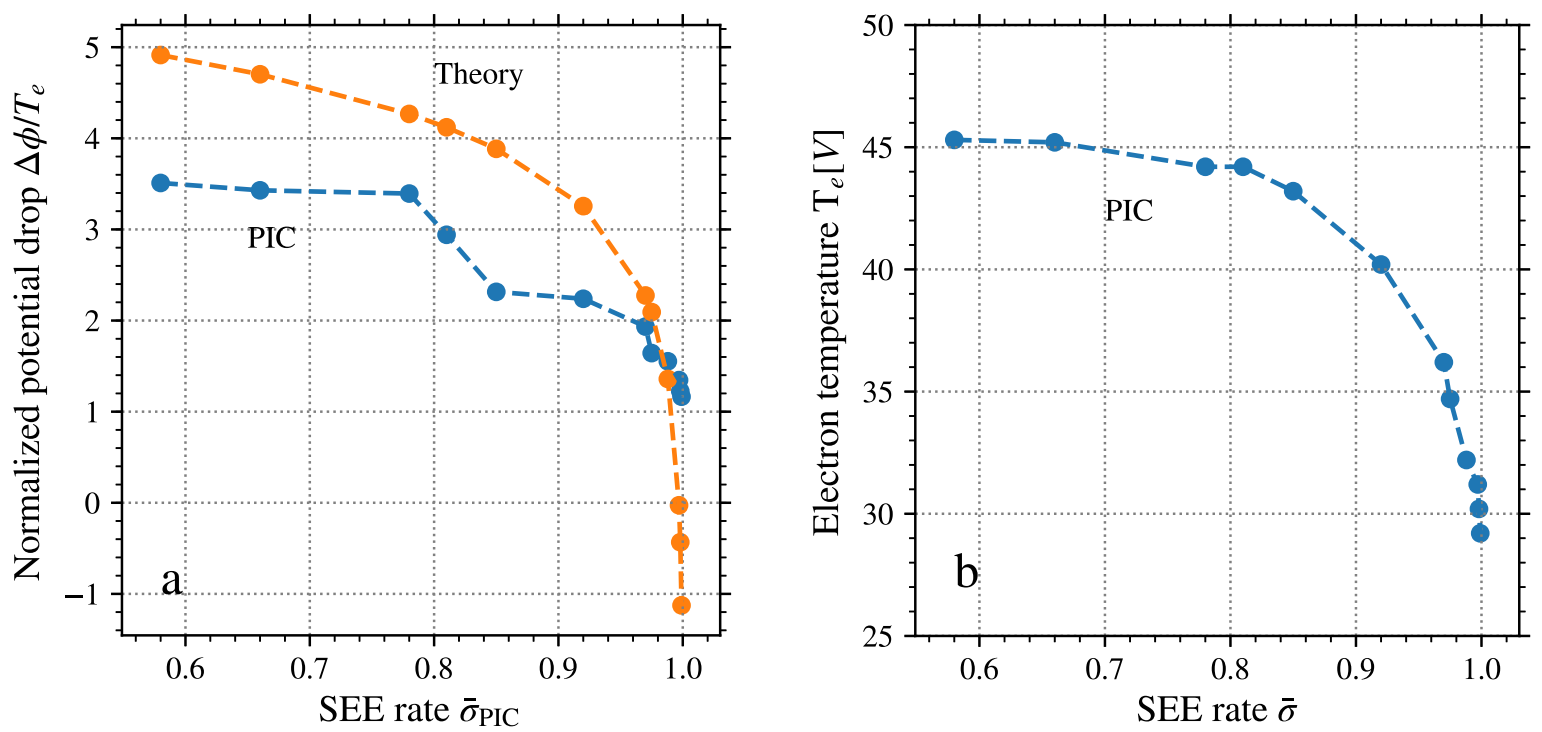

Figure 5. (a) Plasma potential drop to the wall normalized by the electron temperature $\Delta \phi / T_{e}$ as a function of the SEE rate $\bar{\sigma}_{\mathrm{PIC}}$ for the PIC simulations and the theory of equation (6); (b) the electron temperature measured in the PIC simulation as a function of the SEE rate.
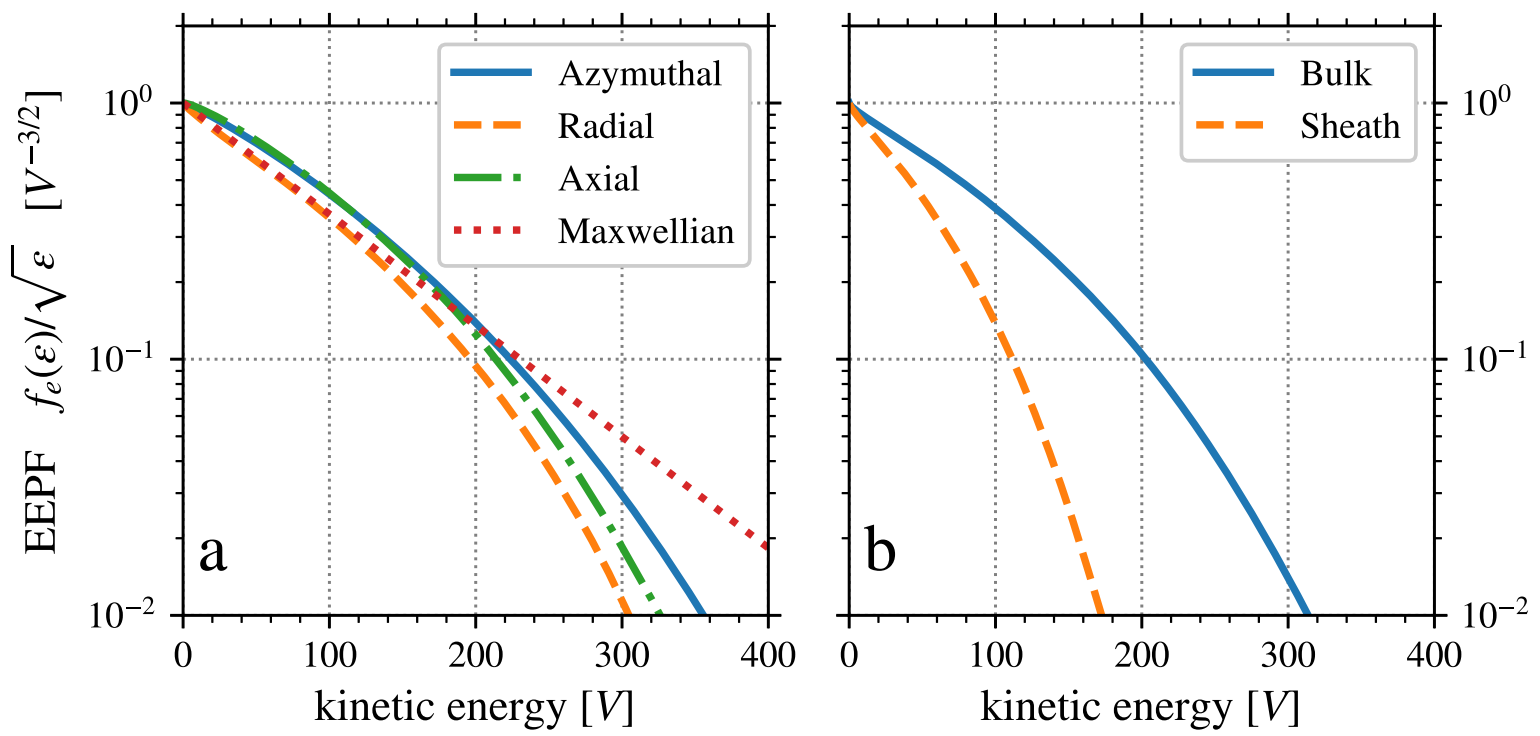

Figure 6. Electron energy probability functions for $\epsilon^{*}=200 \mathrm{~V}$. (a) in the bulk of the plasma, compared with Maxwellian probability of the same temperature $\left(T_{e}=49 \mathrm{~V}\right)$ in red dotted line, and (b) for the bulk and the sheath populations.

simulation results. An assumption used in section 2 is that the sheath is monotonic and at a steady state. For high SEE rate ( i.e. $\epsilon^{*}<40 \mathrm{~V}$ ) this hypothesis is not respected, hence differences were expected. However, for low SEE rate this hypothesis is respected. The plasma-wall interaction model of section 2 uses two other main hypotheses: a Maxwellian distribution of the electrons, and an isothermal plasma. Both hypotheses will be confronted to the kinetic simulation data in the next sections.

\subsection{Sheath electron energy distribution}

Using the kinetic information of the PIC simulations, we present in figure 6 the mean electron energy probability functions (EEPF) in the case $\epsilon^{*}=200 \mathrm{~V}$. Figure 6(a) shows the projections of the EEPF in the bulk of the simulations along the three directions. These projections are compared to the Maxwellian probability function of the same kinetic temperature calculated using equation (7). Figure 6(b) shows the total EEPF for both the bulk and the sheath populations. The sheath length is defined as the location where the ions reach the Bohm speed, and is about $0.4 \mathrm{~mm}$.

We see in figure 6(a) that the electron distribution function is not Maxwellian. In particular the high energy tails are depleted. In order to evaluate the effect of the non-Maxwellian EEPF, we numerically integrate the EEPF from the PIC data using equation (3). The results (not shown) do not differ significantly from the Maxwellian values of equation (4). Hence, we can conclude that even if the Maxwellian hypothesis is not respected in the PIC simulations, it is not enough to explain the differences observed in figure 4. 


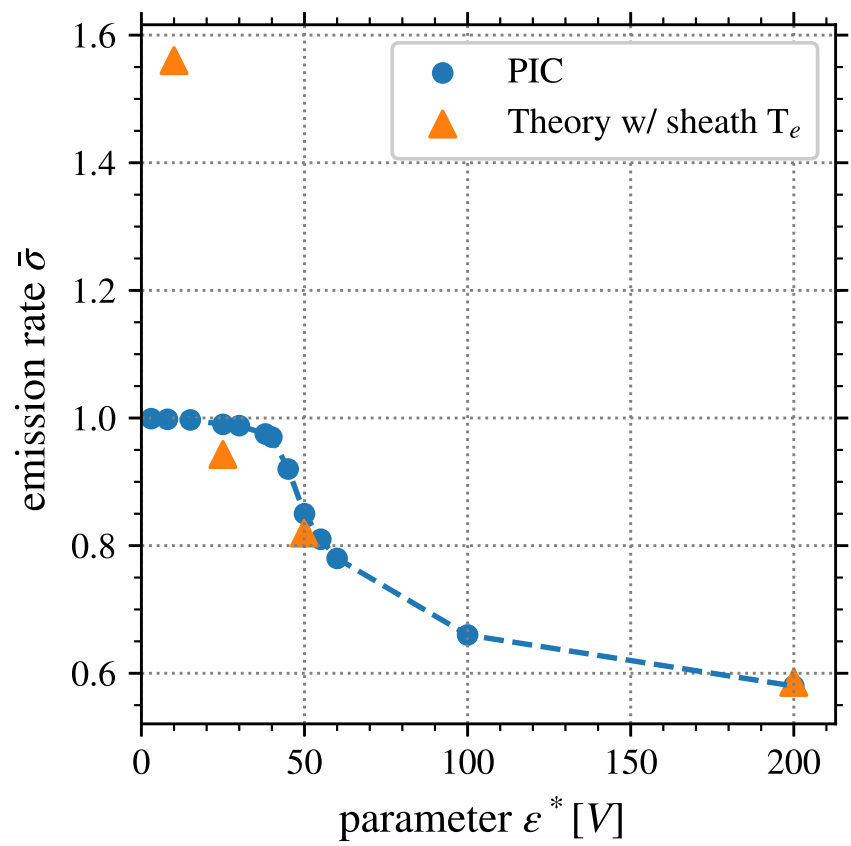

Figure 7. SEE rates as a function of the re-emission parameter $\epsilon^{*}$. Comparison of $\bar{\sigma}_{\text {PIC }}$ derived from the PIC simulations and $\bar{\sigma}_{\text {Maxw }}$, the Maxwellian theory of equation (4) with the sheath temperature.

Figure 6(b) presents the EEPF for the bulk population as well as for the sheath population. We can see that the sheath population is colder than the population at the centre, which could explain the difference of figure 4 . This effect is assessed in the next section.

Surprisingly, we do not observe in figure 6 secondary electron beams in the radial direction, unlike previous observations of Sydorenko et al [45] with a 1D model or Hèron and Adam [33] with a 2D model. On the other hand, we do observe SEE beams when we artificially remove the ECDI by forcing $E_{y}=0 \mathrm{~V} \mathrm{~m}^{-1}$ [40]. Hence, it seems that the ECDI, when simulated in 2D, quickly thermalizes the secondary electrons emitted from the walls. In addition, we can see in figure 6(a) that the radial EEPF is close to the EEPF in the other directions. For instance, for $\epsilon^{*}=200 \mathrm{~V}$ we measure $T_{e \|}=41.5 \mathrm{~V}$ while $T_{e \perp}=49 \mathrm{~V}$. The cause of the difference with Hèron and Adam [33] is not yet clear, but there the results were only obtained at early times where saturation of the instability has not necessarily been reached, and no electron loss in the axial direction was accounted for. It is possible that at steady state, the electrons in our simulations have had sufficient time to become more isotropic. The energy transfer from the axial and azimuthal directions to the radial direction is not clearly understood yet [46], but we believe that it is due to the instability.

\subsection{Corrected SEE rate}

We can calculate the sheath electron temperature using equation (7) from the EEPF obtained in the sheath as shown in figure 6(b). Figure 7 shows the values of the SEE rate measured in the PIC simulation compared to the values obtained by equation (4) using this time the sheath electron temperature. As the calculation of the EEPF is very computationally costly, we have calculated its value for only 4 simulations corresponding to $\epsilon^{*}=\{10,25,50,200\} \mathrm{V}$.

We observe from figure 7 that using the sheath electron temperature in equation (4), the SEE rate is well predicted (error less than 5\%), except for $\epsilon^{*}=10 \mathrm{~V}$ where a large error is observed.

As previously discussed, the case $\epsilon^{*}=10 \mathrm{~V}$ presents a very high SEE rate which leads to a potential well close to the wall. Consequently, some secondary electrons emitted at low energy would be reflected back to the wall. Hence, since equation (4) does not take into account this local effect, it is not surprising that the SEE rate calculated using the mean electron temperature in the sheath is too high.

To summarise, figure 7 shows that when the sheath potential profile is monotonic, the SEE rate can be well predicted by equation (4) using $T_{e}$ in the sheath, which is lower than in the centre of the domain. The energy flux inducing the SEE is not well described if we use the electron temperature of the bulk as in the isothermal sheath model. This explains the overestimations of $\bar{\sigma}$ and $\Delta \phi$ presented in figures 4 and 5. Hence, the isothermal hypothesis used in the sheath model of section 2.3 is denied by the PIC simulations.

This is an important result because the sheath model described in section 2.3 is used in several fluid simulations [32, 36, 37]. Hence, further work is needed to develop an improved, non-isothermal, sheath model in order to better account for the SEE in fluid simulations and theoretical models.

\subsection{Sheath oscillations}

The evolution of the SEE rate averaged over time and the whole computational domain as a function of $\epsilon^{*}$ (figure 4) is smooth, decreasing monotonically from $\bar{\sigma} \sim 1$ to $\sigma_{0}$ when $\epsilon^{*}$ increases. To go further in the analysis, we have also studied the time evolution of $\bar{\sigma}$ for various values of $\epsilon^{*}$.

Figure 8 presents the temporal evolution of the space average $\bar{\sigma}_{\text {PIC }}$ for three different values of $\epsilon^{*}$, corresponding to three different regimes we have identified. In regimes $\mathbf{I}$ and III, $\bar{\sigma}_{\text {PIC }}$ reaches a steady state after a few microseconds. Regime $\mathbf{I}$, with low $\epsilon^{*}$, is characterized by a saturation of $\bar{\sigma}$ at a value between $\bar{\sigma}_{\text {cr }}$ and 1, which leads to a non-monotonic potential profile. Regime III, for high $\epsilon^{*}$, is characterized by a steady state with a SEE rate lower than $\bar{\sigma}_{\mathrm{cr}}$. This is the standard regime described in section 2. The transition between these two stable regimes (monotonic and non-monotonic sheath) passes by regime II, an oscillating mode between the two stable regimes. As shown in figure 9, regime II only exists in a narrow range of $\epsilon^{*}$. The oscillations of regime II are shown in figure 8 up to $10 \mu$ s but have been observed for more than $40 \mu \mathrm{s}$. Note that regimes I and III in figure 8 are obtained for $\epsilon^{*}=38 \mathrm{~V}$ and $\epsilon^{*}=50 \mathrm{~V}$ respectively, i.e. near the boundary of the unstable window (see figure 9). Consequently, we observe a few oscillations before the steady-state is reached, as these cases are close to the bifurcation to the relaxation oscillation regime. 


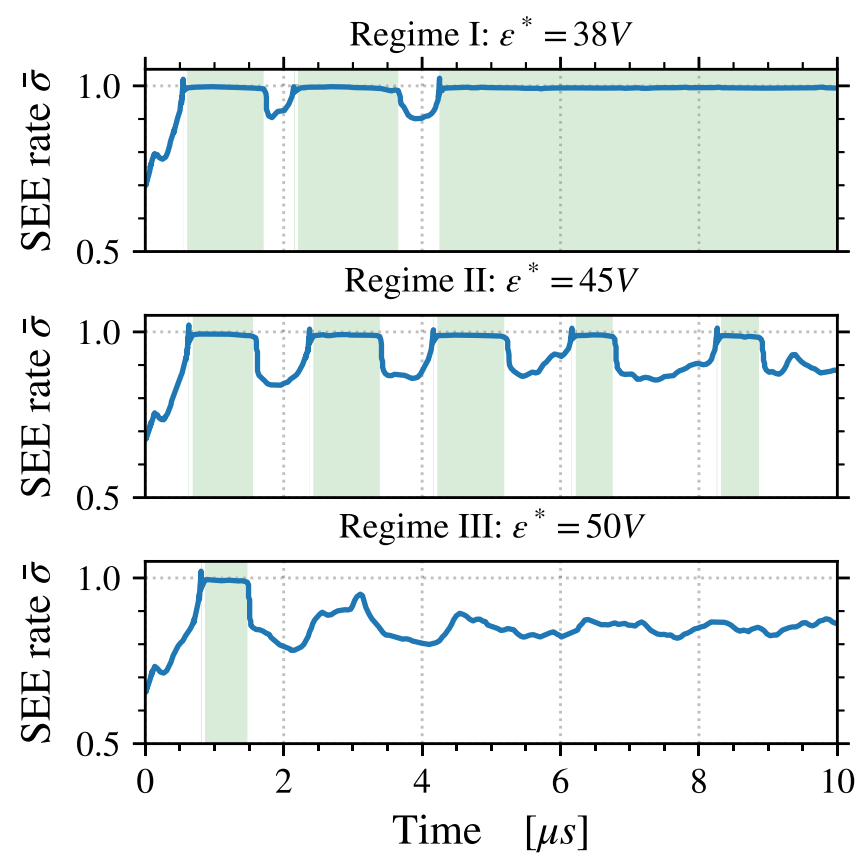

Figure 8. Evolution as a function of time of the averaged SEE rate $\bar{\sigma}$ in the 3 regimes observed, 2 stable and one with oscillations. The light green zones correspond to the periods when $\bar{\sigma}>\bar{\sigma}_{\mathrm{cr}}$.

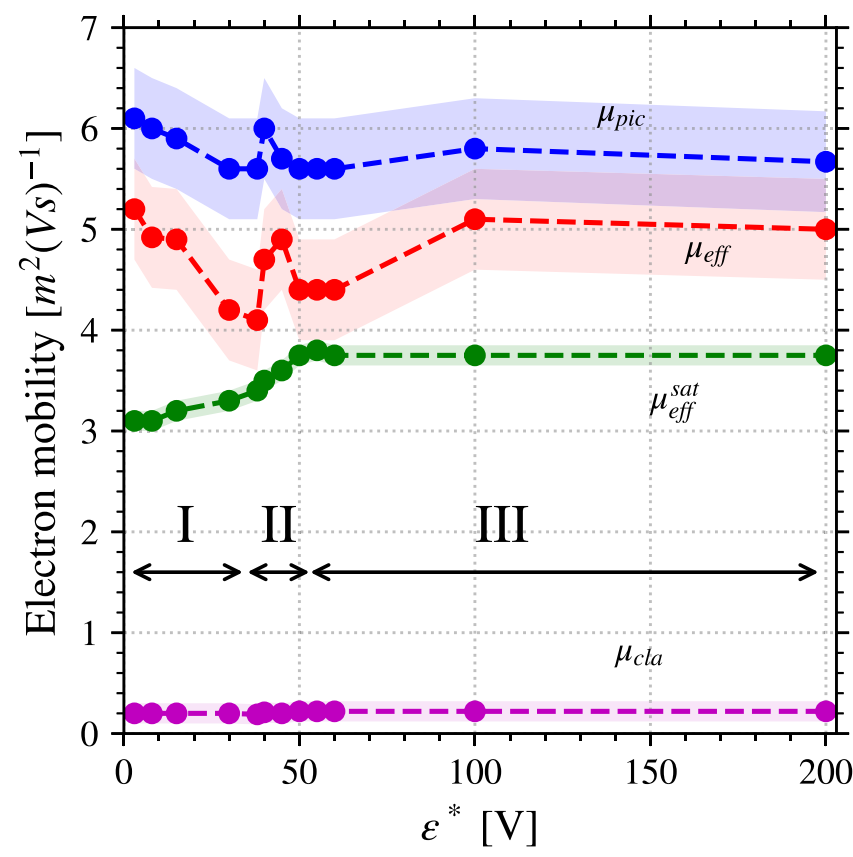

Figure 9. Electron mobility as a function of the parameter $\epsilon^{*} . \mu_{\text {PIC }}$ in blue is directly measured in the PIC simulations, while $\mu_{\text {classical }}, \mu_{\text {eff }}$ and $\mu_{\text {eff }}^{\text {sat }}$ respectively in purple, red and green, are calculated respectively with equations (12), (16) and (11). Three regimes are identified by I, II and III.

Similar oscillations have been observed in the 1D simulation of Sydorenko et al [44]. The oscillations occur because the mean electron energy evolves slowly during the two stable states: when $\bar{\sigma}>\bar{\sigma}_{\text {cr }}$ the electron heat flux to the wall is higher, hence decreasing the electron energy. When the mean electron energy is lower than some threshold, the sheath changes quickly to a stable state where the electron heat flux to the wall is lower, hence increasing the mean electron energy. When the electron energy passes another threshold, the sheath shifts to the first state. However, a detailed theory of the relaxation oscillations needs to be done.

\section{Electron enhanced mobility}

Figure 9 presents the evolution, as a function of $\epsilon^{*}$, of the average value of the measured mobility in the simulation $\mu_{\mathrm{PIC}}$ as well as the effective mobility $\mu_{\text {eff }}$, the saturated estimate $\mu_{\mathrm{eff}}^{\mathrm{sat}}$, and the classical mobility $\mu_{\text {classical }}$, from equations (11), (12) and (16) respectively. The average is the same as the one used in figures 4 and 5, i.e. an average both in time and space.

We observe in figure 9 that $\mu_{\mathrm{PIC}}, \mu_{\mathrm{eff}}$ and $\mu_{\mathrm{eff}}^{\text {sat }}$ are all together varying between 3 and $6 \mathrm{~m}^{2}(\mathrm{Vs})^{-1}$ for $\epsilon^{*}$ varying between 4 and $200 \mathrm{~V}$ and are much higher than $\mu_{\text {classical }} \cdot \mu_{\text {PIC }}$ does not evolve much with the SEE rate, even for very high re-emission rate, i.e. very low $\epsilon^{*}$ values. On the other hand, $\mu_{\mathrm{eff}}^{\text {sat }}$ decreases slightly when $\epsilon^{*}$ the re-emission rate decreases from $40 \mathrm{~V}$ to lower values, which increases the discrepancy with $\mu_{\text {PIC }}$. However, $\mu_{\text {eff }}^{\text {sat }}$ still provides a reasonable approximation of the electron enhanced mobility even with very high SEE rates.

Figure 10 presents the radial profiles of the mobility measured in the PIC simulations $\mu_{\mathrm{PIC}}$ on the left, and on the right the mobility predicted due to the ECDI $\mu_{\text {eff }}$ for three values of $\epsilon^{*}$, obtained by averaging the mobility in the azimuthal direction during $5 \mu \mathrm{s}$ of the simulation. The three chosen values correspond to the three regimes observed in figures 9 and 8: high SEE (regime I), low SEE (regime III) and the transition regime (regime II). The results obtained without SEE are also shown.

We observe in figure 10 that the mobility measured in the centre decreases by $20 \%$ as $\epsilon^{*}$ decreases, i.e. the SEE rate increases. This observation is consistent with the evolution of $\mu_{\text {eff }}$ as well as with the evolution of $\mu_{\text {eff }}^{\text {sat }}$ in figure 9. This is due to the decrease of the electron temperature due to the SEE as seen in figure 5(b). We note in passing that the above results are in apparent disagreement with the observations obtained in Hèron and Adam [33], where anomalous transport was found to be higher at lower values of $\epsilon^{*}$ (see for example figure 24 in Hèron and Adam [33]. However, since no axial loss mechanism was included in those simulations, a steady state solution was not reached, and as shown in figure 23 in Hèron and Adam [33], the anomalous transport rate is still increasing with time for large values of $\epsilon^{*}$; even at the end of the simulation. Since the SEE rate decreases with an increased $\epsilon^{*}$, this results in less electron cooling which causes the bulk electron temperature to increase (this would be further exacerbated by a lack of any axial loss mechanism). By considering the linear growth rate of the most dominant mode of the instability [15], it is found that a higher electron temperature reduces the growth rate. Thus, a longer time is needed to reach a given instability amplitude. Since the instability amplitude largely determines the level of anomalous transport, the difference of our results discussed above with those 

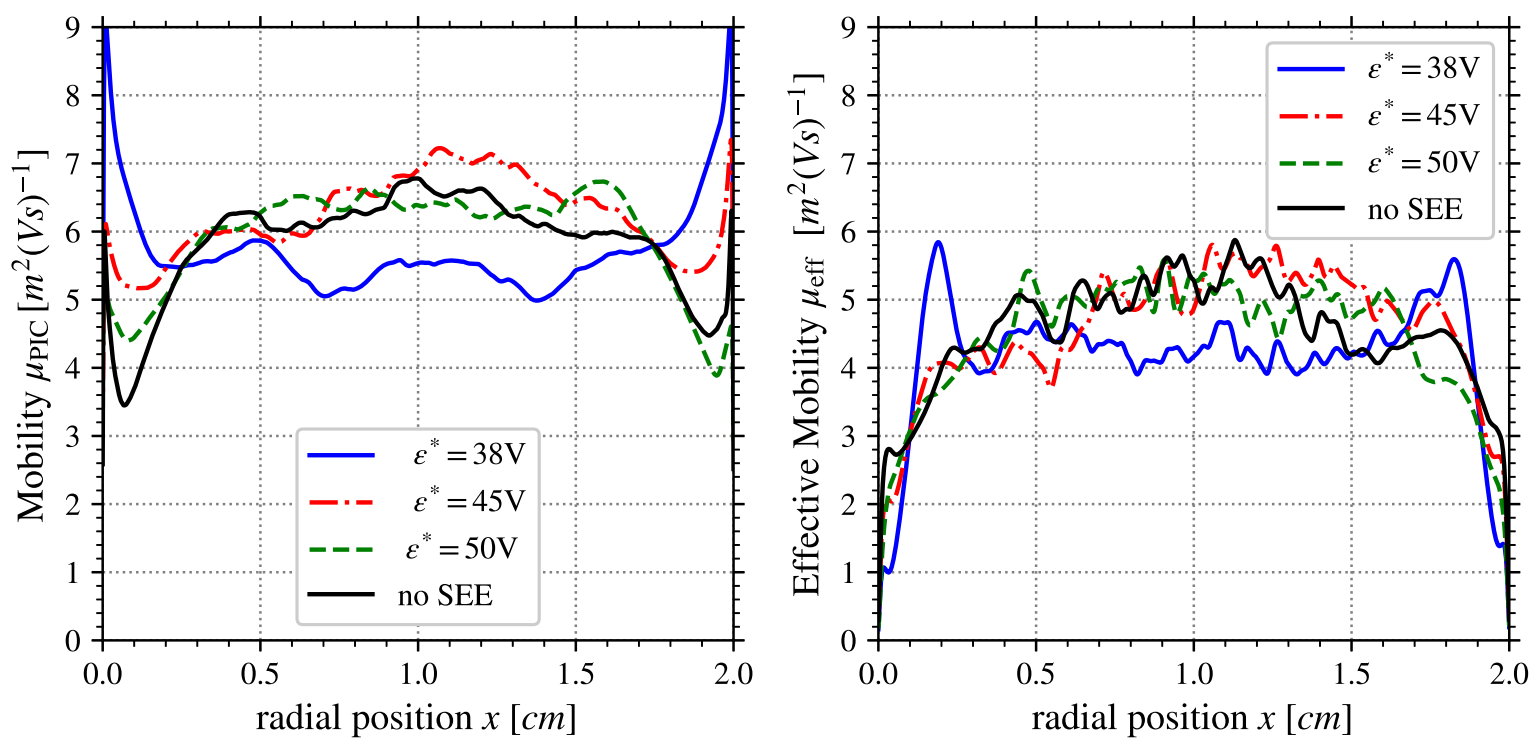

Figure 10. Radial profiles of: (left) the measured mobility in the simulation $\mu_{\mathrm{PIC}}$; (right) the effective mobility in the simulation $\mu_{\text {eff }}$ from equation (12), bothaveraged in the azimuthal direction and in time on $5 \mu \mathrm{s}$.

in Hèron and Adam [33] may only be apparent, and related to the specific time chosen in Hèron and Adam [33] to make the observations. If the simulations in Hèron and Adam [33] could have been run for longer times, it is possible that the anomalous transport rate would have saturated and that similar conclusions to our results above would have been obtained.

On the other hand, the mobility close to the wall, also referred to as near-wall mobility in the literature, increases by almost a factor of 2 with the SEE rate. This near-wall mobility is not observed on the effective mobility shown in figure 10, confirming that it is due to SEE.

These observations of figures 9 and 10 show that when the SEE rate increases, the electron axial mobility in the centre decreases slightly, while the near-wall mobility increases. As a result, even if the SEE rate do vary significantly, the overall mobility measured in the simulations, which takes into account both bulk and near-wall mobilities, is almost constant.

\section{Conclusion}

In this article a study of the effects of SEE from the walls of an $\mathbf{E} \times \mathbf{B}$ discharge were presented. It was observed that the anomalous electron cross-field transport in the bulk plasma decreases by $20 \%$ as the effective SEE coefficient increases. This appears to occur as a direct consequence of electron cooling, and demonstrates a coupling between SEE and the electron drift instability. The observation of reduced electron transport in the plasma bulk due to a lower electron temperature is in good agreement with the kinetic theory predictions of Lafleur et al [15]. However, when the SEE coefficient increases from 0 to almost 1 , the near-wall mobility increases almost by a factor 2 . As a result, the total mobility in the simulations is found to remain almost constant. Consequently, in our simulations the main effect of SEE is to decrease the electron temperature by both decreasing the potential drop at the wall, and through the injection of cold electrons into the discharge. Overall though the dominant contribution to anomalous electron transport is caused by the electron drift instability.

The plasma characteristics in the simulations have been compared to a typical plasma sheath model that accounts for SEE. The effective SEE coefficient observed in the simulations appears much lower than the predicted value from this model, even though no electron beams have been observed, contrary to Sydorenko et al [45]. Hèron and Adam [33] also observed such beams, but do not compare the measured SEE rate to a theoretical value. However, we observed that the electron temperature is lower in the sheath than in the bulk plasma, which when accounted for, correctly explains the discrepancies observed in the SEE coefficient and potential drop at the wall. Further work is needed to develop an improved, non-isothermal, sheath model in order to better account for plasma-surface interactions in fluid simulations and theoretical models.

Three regimes have been identified when varying the effective SEE coefficient. For low SEE rates, the plasma sheath at the walls has a typical monotonic spatial profile. This region is stable. For high SEE rates, a potential well is observed to form near the wall due to space charge effects associated with intense electron emission. This saturated regime is also found to be stable. However, for intermediate SEE rates, an oscillatory behaviour is observed where the potential profile changes between those for a standard monotonic sheath, and the saturated sheath. The main trigger for this oscillatory behaviour is not yet understood, but this regime appears similar to the relaxation sheath oscillations observed before by Sydorenko et al [44].

In order to improve the realism of the simulation, two limitations of the current study should be addressed: (1) the 
radial walls are conducting instead of insulating (although they still float electrically like an insulator), (2) the simulation domain neglects the curvature of the thruster channel. Both of these points are expected to alter the equilibrium values [39], but not necessarily the overall conclusion on the electron transport and the SEE effects.

\section{Acknowledgments}

The authors would like to thank Franois Pechereau, Abdoul Wahid C Mainassara, Thomas Charoy, Roberto Martorelli and Stephan Zurbach for many useful discussions, as well as for their help and support in the development of the LPPic simulation code. VC and AT acknowledge financial and technical support from a Safran Aircraft Engines doctoral research award. This work has been partially funded byANR ( ${ }^{\circ}$ ANR-16-CHIN-003-01) and Safran Aircraft Engines with the project POSEIDON. The authors were granted access to the HPC resources of CINES (under the allocation A0020510092 and A0040510092 made by GENCI) and of CERFACS.

\section{Appendix}

The exact expression of the SEE rate using equations (1)-(3) can be found to be:

$$
\begin{aligned}
\bar{\sigma}_{\text {Maxw }}\left(T_{e}\right)= & \sigma_{0}+\frac{1-\sigma_{0}}{\epsilon^{*}} 2 T_{e} \\
& +\left(\sigma_{\max }-\sigma_{0}\right) \mathrm{e}^{-\alpha^{2}}\left(\alpha^{2}+1\right) \\
& +T_{e} \frac{\sigma_{0}-1}{\epsilon^{*}} \mathrm{e}^{-\alpha^{2}}\left(\alpha^{2}\left(\alpha^{2}+2\right)+2\right)
\end{aligned}
$$

with

$$
\alpha^{2}=\frac{1}{T_{e}}\left(\frac{\sigma_{\max }-\sigma_{0}}{1-\sigma_{0}} \epsilon^{*}\right)^{2} .
$$

We can see from equation (17) that if $\alpha^{2} \gg 1$ then $\bar{\sigma}_{\text {Maxw }}\left(\mathrm{T}_{e}\right) \simeq \sigma_{0}+\frac{1-\sigma_{0}}{\epsilon^{*}} 2 \mathrm{~T}_{e}$. In the case $\epsilon^{*}=200 \mathrm{~V}, \alpha \simeq 2 \times 10^{4}$.

\section{ORCID iDs}

Antoine Tavant (iD https://orcid.org/0000-0003-0010-8073 Romain Lucken (1D https://orcid.org/0000-0002-2363-0844 Trevor Lafleur (1D https://orcid.org/0000-0002-9926-2187 Anne Bourdon (ib https://orcid.org/0000-0003-3662-4651

\section{References}

[1] Mazouffre S 2016 Electric propulsion for satellites and spacecrafts: established technologies and novel approaches Plasma Sources Sci. Technol. 253
[2] Goebel D M and Katz I 2008 Fundamentals of Electric Propulsion: Ion and Hall Thrusters (New York: Wiley)

[3] Morozov A I and Savelyev V V 2000 Reviews of Plasma Physics ed B B Kadomtsev and V D Shafranov (New York: Springer Science)

[4] Adam J C, Héron A and Laval G 2004 Study of stationary plasma thrusters using two-dimensional fully kinetic simulations Phys. Plasmas 11295

[5] Boeuf J P 2017 Tutorial: physics and modeling of Hall thrusters J. Appl. Phys. 1211

[6] Meezan N B, Hargus W A Jr. and Cappelli M A 2001 Anomalous electron mobility in a coaxial Hall discharge plasma Phys. Rev. E 63026410

[7] Garrigues L, Pérez-Luna J, Lo J, Hagelaar G J M, Boeuf J P and Mazouffre S 2009 Empirical electron crossfield mobility in a Hall effect thruster Appl. Phys. Lett. 95 141501

[8] Adam J C et al 2008 simulation, and diagnostics of Hall effect thrusters Plasma Phys. Control. Fusion 24124041

[9] Yoshikawa S and Rose D J 1962 Anomalous diffusion of a plasma across a magnetic field Phys. Fluids 5334

[10] Janes G S and Lowder R S 1966 Anomalous electron diffusion and ion acceleration in a low density plasma Phys. Fluids 9115

[11] Choueiri E Y 2001 Fundamental difference between the two Hall thruster variants Phys. Plasmas 81411

[12] Smith A W and Cappelli M A 2009 Time and space-correlated plasma potential measurements in the near field of a coaxial Hall plasma discharge Phys. Plasmas 16073504

[13] Scharfe M K, Gascon N, Cappelli M A and Fernandez E 2006 Comparison of hybrid Hall thruster model to experimental measurements Phys. Plasmas 13083505

[14] Croes V, Lafleur T, Bonaventura Z, Bourdon A and Chabert P 2017 2D particle-in-cell simulations of the electron drift instability and associated anomalous electron transport in hall-effect thrusters Plasma Sources Sci. Technol. 263

[15] Lafleur T, Baalrud S D and Chabert P 2016 Theory for the anomalous electron transport in Hall effect thrusters: II. Kinetic model Phys. Plasmas 23053503

[16] Lafleur T and Chabert P 2017 The role of instability-enhanced friction on 'anomalous' electron and ion transport in Halleffect thrusters Plasma Sources Sci. Technol. 271

[17] Lafleur T, Baalrud S D and Chabert P 2016 Theory for the anomalous electron transport in Hall effect thrusters: I. Insights from particle-in-cell simulations Phys. Plasmas 23 053502

[18] Gascon N, Dudeck M and Barral S 2003 Wall material effects in stationary plasma thrusters: I. Parametric studies of an SPT-100 Phys. Plasmas 104123

[19] Zhurin V V, Kaufman H R and Robinson R S 1999 Physics of closed drift thrusters Plasma Sources Sci. Technol. 81

[20] Kaganovich I D, Raitses Y, Sydorenko D and Smolyakov A 2007 Kinetic effects in a Hall thruster discharge Phys. Plasmas 14057104

[21] Sydorenko D, Smolyakov A I, Kaganovich I D and Raitses Y 2008 Electron kinetic effects and beam related instabilities in Hall thrusters Phys. Plasmas 15053506

[22] Hirakawa M 1997 Electron transport mechanism in a Hall thruster IEPC-97-021

[23] Boniface C, Garrigues L, Hagelaar G J M, Boeuf J P, Gawron D and Mazouffre S 2006 Anomalous cross-field electron transport in a Hall thruster Appl. Phys. Lett. 89 161503

[24] Hagelaar G J M, Bareilles J, Garrigues L and Boeuf J P 2003 Role of anomalous electron transport in a stationary plasma thruster simulation J. Appl. Phys. 9367

[25] Meezan N B and Capelli M A 2002 Kinetic study of wall collisions in a Coaxial Hall discharge Phys. Rev. E 66 036401 
[26] Parra F I, Ahedo E, Fife J M and Martinez-Sanchez M 2006 A two-dimensional hybrid model of the hall thruster discharge J. Appl. Phys. 100023304

[27] Smirnov A N, Raitses Y and Fisch N J 2006 Electron crossfield transport in a miniaturized cylindrical Hall thruster IEEE Trans. Plasma Sci. 34132

[28] Mazouffre S, Tsikata S and Vaudolon J 2014 Development and experimental characterization of a wall-less Hall thruster J. Appl. Phys. 11624

[29] Mikellides I G, Katz I, Hofer R R and Goebel D M 2012 Magnetic shielding of walls from the unmagnetized ion beam in a Hall thruster Appl. Phys. Lett. 1022

[30] Hobbs G D and Wesson J A 1967 Heat flow through a Langmuir sheath in the presence of electron emission Plasma Phys. 91

[31] Sydorenko D, Smolyakov A I, Kaganovich I D and Raitses Y 2005 Kinetic simulation of secondary electron emission effects in Hall thrusters Phys. Plasmas 13102111

[32] Barral S, Makowski K, Peradznski Z, Gascon N and Dudeck M 2003 Wall material effects in stationary plasma thrusters: II. Near-wall and in-wall conductivity Phys. Plasmas 104137

[33] Héron A and Adam J C 2013 Anomalous conductivity in Hall thrusters: effects of the non-linear coupling of the electroncyclotron drift instability with secondary electron emission of the walls Phys. Plasmas 20082313

[34] Taccogna F, Longo S, Capitelli M and Schneider R 2009 Anomalous transport induced by sheath instability in Hall effect thrusters Appl. Phys. Lett. 94251502

[35] Garrigues L, Hagelaar G, Boeuf J P, Raitses Y, Smirnov A and Fisch N J 2008 Simulations of a miniaturized cylindrical hall thruster IEEE Trans. Plasma Sci. 365

[36] Ahedo E 2002 Presheath/sheath model with secondary electron emission from two parallel walls Phys. Plasma 910
[37] Ahedo E and Parra F I 2005 Partial trapping of secondaryelectron emission in a Hall thruster plasma Phys. Plasma 127

[38] Langendorf S and Walker M 2015 Effect of secondary electron emission on the plasma sheath Phys. Plasma 223

[39] Domínguez-Vázquez A, Taccogna F and Ahedo E 2018 Particle modeling of radial electron dynamics in a controlled discharge of a Hall thruster Plasma Sources Sci. Technol. 27 064006

[40] Croes V 2017 Modelisation bidimensionnelle de la decharge plasma dans un propulseur de Hall PhD Thesis Ecole Polytechnique

[41] Birdsall C K and Langdon A B 1985 Plasma Physics via Computer Simulation (New York: McGraw-Hill)

[42] Boeuf J P 2014 Rotating structures in low temperature magnetized plasmas-insight from particle simulations Front. Phys. 274

[43] Smirnov A N, Raitses Y and Fisch N J 2004 Electron crossfield transport in a low power cylindrical Hall thruster Phys. Plasmas 114922

[44] Sydorenko D, Kaganovich I D, Raitses Y and Smolyakov A I 2009 Breakdown of a space charge limited regime of a sheath in a weakly collisional plasma bounded by walls with secondary electron emission Phys. Rev. Lett. 103145004

[45] Sydorenko D, Smolyakov A, Kaganovich I and Raitses Y 2006 Kinetic simulation of secondary electron emission effects in Hall thrusters Phys. Plasma 13014501

[46] Janhunen S, Smolyakov A, Sydorenko D, Jimenez M, Kaganovich I and Raitses Y 2018 Evolution of the electron cyclotron drift instability in two-dimensions Phys. Plasma 25082308 